Research Article

\title{
Thermodynamic Product Relations for Generalized Regular Black Hole
}

\author{
Parthapratim Pradhan \\ Department of Physics, Vivekananda Satavarshiki Mahavidyalaya (Affiliated to Vidyasagar University), Manikpara, \\ West Midnapore, West Bengal 721513, India
}

Correspondence should be addressed to Parthapratim Pradhan; pppradhan77@gmail.com

Received 14 April 2016; Accepted 3 July 2016

Academic Editor: Masoud S. Rad

Copyright (c) 2016 Parthapratim Pradhan. This is an open access article distributed under the Creative Commons Attribution License, which permits unrestricted use, distribution, and reproduction in any medium, provided the original work is properly cited. The publication of this article was funded by SCOAP ${ }^{3}$.

We derive thermodynamic product relations for four-parametric regular black hole $(\mathrm{BH})$ solutions of the Einstein equations coupled with a nonlinear electrodynamics source. The four parameters can be described by the mass $(m)$, charge $(q)$, dipole moment $(\alpha)$, and quadrupole moment $(\beta)$, respectively. We study its complete thermodynamics. We compute different thermodynamic products, that is, area product, $\mathrm{BH}$ temperature product, specific heat product, and Komar energy product, respectively. Furthermore, we show some complicated function of horizon areas that is indeed mass-independent and could turn out to be universal.

\section{Introduction}

The thermodynamic product for Reissner Nordstrøm (RN) $\mathrm{BH}$, Kerr BH, and Kerr-Newman $(\mathrm{KN}) \mathrm{BH}[1]$ has been examined; a simple area product is sufficient to draw a conclusion that the product of area (or entropy) is a universal quantity. The universal term is used here to describe when the product of any thermodynamic quantities is simply massindependent. Alternatively mass-dependent thermodynamic quantities imply that they are not universal quantity. This is strictly followed throughout the work.

In case of RN-AdS [2], Kerr-AdS, and KN-AdS BH [3] one can not find a simple area product relation; instead one could find a complicated function of event horizon $\left(\mathscr{H}^{+}\right)$area and Cauchy horizon area $\left(\mathscr{H}^{-}\right)$that might be universal. Very recently, we derived for a regular Ayón-Beato and García BH (ABG) [4] the function of $\mathscr{H}^{+}$area and $\mathscr{H}^{-}$area is

$$
f\left(\mathscr{A}_{+}, \mathscr{A}_{-}\right)=384 \pi^{3} q^{6},
$$

where the function should read

$$
\begin{aligned}
& f\left(\mathscr{A}_{+}, \mathscr{A}_{-}\right)=\mathscr{A}_{+} \mathscr{A}_{-}\left(\mathscr{A}_{+}+\mathscr{A}_{-}\right)+24 \pi q^{2} \mathscr{A}_{+} \mathscr{A}_{-} \\
& -256 \pi^{4} q^{8}\left(\frac{\mathscr{A}_{+}+\mathscr{A}_{-}}{\mathscr{A}_{+} \mathscr{A}_{-}}\right)
\end{aligned}
$$

$$
\begin{aligned}
& -\frac{\mathscr{A}_{+} \mathscr{A}_{+}}{\mathscr{A}_{+}+\mathscr{A}_{+}+4 \pi q^{2}}\left[\left(\mathscr{A}_{+}+\mathscr{A}_{-}\right)^{2}\right. \\
& +24 \pi q^{2}\left(\mathscr{A}_{+}+\mathscr{A}_{-}\right)-\mathscr{A}_{+} \mathscr{A}_{-}-\frac{256 \pi^{4} q^{8}}{\mathscr{A}_{+} \mathscr{A}_{-}} \\
& \left.+176 \pi^{2} q^{4}\right] .
\end{aligned}
$$

It indicates a very complicated function of horizons area that turns out to be universal. But it is not a simple area product of horizon radii as in $\mathrm{RN} \mathrm{BH}, \mathrm{Kerr} \mathrm{BH}$, and $\mathrm{KN} \mathrm{BH}$. This has been very popular topic in recent years in the GR (General Relativity) community [1] as well as in the String community [5] (see also [6-10]). These universal relations are particularly interesting because they could hold in more general situations like when a $\mathrm{BH}$ space-time is perturbed by surrounding matter. For example, $\mathrm{KN}$ BH surrounded by a ring of matter the universal relation does indeed holds [1].

Recently, Page and Shoom [8] have given a heuristic argument for the universal area product relation of a fourdimensional adiabatically distorted charged rotating $\mathrm{BH}$. They in fact showed that the product of outer horizon area 
and inner horizon area could be expressed in terms of a polynomial function of its charge, angular momenta, and inverse square root of cosmological constant. It has been argued by Cvetič et al. [5] that if the cosmological parameter is quantized, the product of $\mathscr{H}^{+}$area and $\mathscr{H}^{-}$area could provide a "looking glass for probing the microscopy of general BHs".

However, in this work, we would like to evaluate the thermodynamic product formula for a generalized regular (singularity-free) $\mathrm{BH}$ described by the four parameters, namely, $m, q, \alpha$, and $\beta$ [11]. This class of $\mathrm{BH}$ is a solution of Einstein equations coupled with a nonlinear electrodynamics source. We examine complete thermodynamic properties of this $\mathrm{BH}$. We show some complicated function of physical horizon areas that is indeed mass-independent but it is not a simple area product as in Kerr $\mathrm{BH}$ or $\mathrm{KN} \mathrm{BH}$. We also compute the specific heat to examine the thermodynamic stability of the BH. Finally we compute the Komar energy of this $\mathrm{BH}$.

It should be noted that Smarr's mass formula and BoseDadhich identity do not hold for $\alpha=3$ and $\beta=3$ when one has taken into account the nonlinear electrodynamics [1215]. It also should be mentioned that, for some regular $\mathrm{BHs}$ [16], once the entropy is taken to be the Bekenstein-Hawking entropy $[17,18]$ the first law of $\mathrm{BH}$ thermodynamics is no longer established because there is an inconsistency between the conventional 1st law of $\mathrm{BH}$ mechanics and BekensteinHawking area law. The authors [16] also showed the corrected form of the first law of $\mathrm{BH}$ thermodynamics for this class of regular $\mathrm{BHs}$. We should expect that this is also true for regular $\mathrm{ABG} \mathrm{BH}$ and it should be valid for arbitrary values of $\alpha$ and $\beta$.

The plan of the paper is as follows. In Section 2, we have described the basic properties of the generalized regular $\mathrm{BH}$ and computed various thermodynamic properties. Finally, we conclude our discussions in Section 3.

\section{Generalized Regular BH Solution}

The gravitational field around the four-parametric regular $\mathrm{BH}$ solution is described by the metric

$$
d s^{2}=-\mathscr{B}(r) d t^{2}+\frac{d r^{2}}{\mathscr{B}(r)}+r^{2}\left(d \theta^{2}+\sin ^{2} \theta d \phi^{2}\right),
$$

where the function $\mathscr{B}(r)$ is defined by

$$
\mathscr{B}(r)=1-\frac{2 m r^{\alpha-1}}{\left(r^{2}+q^{2}\right)^{\alpha / 2}}+\frac{q^{2} r^{\beta-2}}{\left(r^{2}+q^{2}\right)^{\beta / 2}} .
$$

And the electric field is given by

$$
\begin{aligned}
& \mathscr{E}(r)=q\left[\frac{m \alpha\left\{5 r^{2}-(\alpha-3) q^{2}\right\} r^{\alpha-1}}{\left(r^{2}+q^{2}\right)^{\alpha / 2+2}}\right. \\
& \left.+\frac{\left\{4 r^{4}-(7 \beta-8) q^{2} r^{2}+(\beta-1)(\beta-4)^{4}\right\} r^{\beta / 2}}{4\left(r^{2}+q^{2}\right)^{\beta / 2+2}}\right],
\end{aligned}
$$

where the parameters are described previously. This is a class of regular (curvature free) BH solution in GR. The source is nonlinear electrodynamics. In the weak field limits the nonlinear electrodynamics becomes Maxwell field.

In the asymptotic limit, the above solution behaves as

$$
\begin{aligned}
& -g_{t t}=1-\frac{2 m}{r}+\frac{q^{2}}{r^{2}}+\alpha \frac{m q^{2}}{r^{3}}-\beta \frac{q^{4}}{2 r^{4}}+\mathcal{O}\left(\frac{1}{r^{5}}\right), \\
& \mathscr{E}(r)=\frac{q}{r^{2}}+\alpha \frac{5 m}{2 r^{3}}-\beta \frac{9 q^{3}}{4 r^{4}}+\mathcal{O}\left(\frac{1}{r^{5}}\right) .
\end{aligned}
$$

It may be noted that, up to $\mathcal{O}\left(1 / r^{3}\right)$, we recover the charged $\mathrm{BH}$ behavior and the parameters $m$ and $q$ are related to the mass and charges, respectively. From the electric field behavior we can say that $\alpha$ and $\beta$ are associated with the electric dipole moment and electric quadrupole moments, respectively. It also should be noted that, in the limit $\alpha=\beta=$ 0 , we obtain the RN BH and, in the limits $\alpha=3$ and $\beta=4$, we recover the $\mathrm{ABG} B \mathrm{BH}$. This $\mathrm{BH}$ solution can be treated as a generalization of ABG BH [19]. The above metric as well as the scalar invariants $R, R_{a b} R^{a b}, R_{a b c d} R^{a b c d}$ and the electric field are regular everywhere in the space-time. Hence, in this sense it is called a regular $\mathrm{BH}$ in Einstein-Maxwell gravity. The first regular model is discovered by Bardeen [20] in 1968. that is,

The $\mathrm{BH}$ horizons can be obtained by setting $\mathscr{B}(r)=0$;

$$
\begin{gathered}
\left(r^{2}+q^{2}\right)^{(\alpha+\beta) / 2}-2 m r^{\alpha-1}\left(r^{2}+q^{2}\right)^{\beta / 2} \\
+q^{2} r^{\beta-2}\left(r^{2}+q^{2}\right)^{\alpha / 2}=0
\end{gathered}
$$

In this work we restrict our case $\alpha \geq 3$ and $\beta \geq 4$.

Case 1. We have set $\alpha=4$ and $\beta=5$. In this case the horizon equation is found to be

$$
\begin{aligned}
r^{10}- & 2 m r^{9}+\left(4 m^{2}+5 q^{2}\right) r^{8}-6 m q^{2} r^{7} \\
& +\left(4 m^{2} q^{2}+9 q^{4}\right) r^{6}-6 m q^{4} r^{5}+10 q^{6} r^{4} \\
& -2 m q^{6} r^{3}+5 q^{8} r^{2}+q^{10}=0 .
\end{aligned}
$$

To find the roots we apply Vieta's theorem; we find

$$
\begin{aligned}
\sum_{i=1}^{10} r_{i} & =2 m, \\
\sum_{1 \leq i<j \leq 10} r_{i} r_{j} & =4 m^{2}+5 q^{2}, \\
\sum_{1 \leq i<j<k \leq 10} r_{i} r_{j} r_{k} & =6 m q^{2},
\end{aligned}
$$




$$
\begin{aligned}
\sum_{1 \leq i<j<k<l \leq 10} r_{i} r_{j} r_{k} r_{l} & =4 m^{2} q^{2}+9 q^{4}, \\
\sum_{1 \leq i<j<k<l<p \leq 10} r_{i} r_{j} r_{k} r_{l} r_{p} & =6 m q^{4}, \\
\sum_{1 \leq i<j<k<l<p<s \leq 10} r_{i} r_{j} r_{k} r_{l} r_{p} r_{s} & =10 q^{6}, \\
\sum_{1 \leq i<j<k<l<p<s<t \leq 10} r_{i} r_{j} r_{k} r_{l} r_{p} r_{s} r_{t} & =2 m q^{6}, \\
\sum_{1 \leq i<j<k<l<p<s<t<u \leq 10} r_{i} r_{j} r_{k} r_{l} r_{p} r_{s} r_{t} r_{u} & =5 q^{8}, \\
\prod_{i=1}^{10} r_{i} & =q^{10} .
\end{aligned}
$$

Eliminating mass parameter we find the following set of equations:

$$
\begin{aligned}
\sum_{1 \leq i<j \leq 10} r_{i} r_{j}-\left(\sum_{i=1}^{10} r_{i}\right)^{2} & =5 q^{2}, \\
\sum_{1 \leq i<j<k \leq 10} r_{i} r_{j} r_{k} & =3 q^{2} \sum_{i=1}^{10} r_{i}, \\
\sum_{1 \leq i<j<k<l \leq 10} r_{i} r_{j} r_{k} r_{l}-q^{2}\left(\sum_{i=1}^{10} r_{i}\right)^{2} & =9 q^{4}, \\
\sum_{1 \leq i<j<k<l<p \leq 10} r_{i} r_{j} r_{k} r_{l} r_{p} & =3 q^{4} \sum_{i=1}^{10} r_{i}, \\
\sum_{1 \leq i<j<k<l<p<s<t \leq 10} r_{i} r_{j} r_{k} r_{l} r_{p} r_{s} r_{t} & =q^{6} \sum_{i=1}^{10} r_{i} .
\end{aligned}
$$

By further elimination in (10), finally we find the following mass-independent relations:

$$
\begin{aligned}
& \sum_{1 \leq i<j<k<l \leq 10} r_{i} r_{j} r_{k} r_{l}-q^{2} \sum_{1 \leq i<j \leq 10} r_{i} r_{j}=4 q^{4}, \\
& =q_{1 \leq i<j<k<l<p<s<t \leq 10}^{2} \sum_{i \leq i<j<k<l<p \leq 10} r_{j} r_{k} r_{l} r_{p} r_{s} r_{t} r_{j} r_{k} r_{l} r_{p}, \\
& \sum_{1 \leq i<j<k<l<p<s \leq 10} r_{i} r_{j} r_{k} r_{l} r_{p} r_{s}=10 q^{6}, \\
& \sum_{1 \leq i<j<k<l<p<s<t<u \leq 10} r_{i} r_{j} r_{k} r_{l} r_{p} r_{s} r_{t} r_{u}=5 q^{8}, \\
& \prod_{i=1}^{10} r_{i}=q^{10} .
\end{aligned}
$$

In terms of area $\mathscr{A}_{i}=4 \pi r_{i}^{2}$, the mass-independent relations are

$$
\begin{aligned}
& \sum_{1 \leq i<j<k<l \leq 10} \sqrt{\mathscr{A}_{i} \mathscr{A}_{j} \mathscr{A}_{k} \mathscr{A}_{l}}-4 \pi q^{2} \sum_{1 \leq i<j \leq 10} \sqrt{\mathscr{A}_{i} \mathscr{A}_{j}} \\
& =\left(8 \pi q^{2}\right)^{2}, \\
& 3 \sum_{1 \leq i<j<k<l<p<s<t \leq 10} \sqrt{\mathscr{A}_{i} \mathscr{A}_{j} \mathscr{A}_{k} \mathscr{A}_{l} \mathscr{A}_{p} \mathscr{A}_{s} \mathscr{A}_{t}} \\
& =4 \pi q^{2} \sum_{1 \leq i<j<k<l<p \leq 10} \sqrt{\mathscr{A}_{i} \mathscr{A}_{j} \mathscr{A}_{k} \mathscr{A}_{l} \mathscr{A}_{p}}, \\
& \sum_{1 \leq i<j<k<l<p<s \leq 10} \sqrt{\mathscr{A}_{i} \mathscr{A}_{j} \mathscr{A}_{k} \mathscr{A}_{l} \mathscr{A}_{p} \mathscr{A}_{s}}=640 \pi^{3} q^{6},
\end{aligned}
$$

$$
\begin{aligned}
& \sum_{1 \leq i<j<k<l<p<s<t<u \leq 10} \sqrt{\mathscr{A}_{i} \mathscr{A}_{j} \mathscr{A}_{k} \mathscr{A}_{l} \mathscr{A}_{p} \mathscr{A}_{s} \mathscr{A}_{t} \mathscr{A}_{u}} \\
& =1280 \pi^{4} q^{8}, \\
& \prod_{i=1}^{10} \sqrt{\mathscr{A}_{i}}=\left(4 \pi q^{2}\right)^{5} .
\end{aligned}
$$

From this relation one can obtain the mass-independent entropy relation by substituting $\mathscr{A}_{i}=4 \mathcal{S}_{i}$. Thus the massindependent complicated function of horizon areas that we have found could turn out to be a universal quantity.

Case 2. Now we have set $\alpha=5$ and $\beta=6$. In this case the horizon equation is given by

$$
\begin{aligned}
r^{12}- & \left(4 m^{2}-8 q^{2}\right) r^{10}-\left(4 m^{2} q^{2}-22 q^{4}\right) r^{8}+26 q^{6} r^{6} \\
& +17 q^{8} r^{4}+6 q^{10} r^{2}+q^{12}=0 .
\end{aligned}
$$

Let us put $r^{2}=x$; then the equation reduces to sixth-order polynomial equation:

$$
\begin{aligned}
x^{6}- & \left(4 m^{2}-8 q^{2}\right) x^{5}-\left(4 m^{2} q^{2}-22 q^{4}\right) x^{4}+26 q^{6} x^{3} \\
+ & 17 q^{8} x^{2}+6 q^{10} x+q^{12}=0 .
\end{aligned}
$$

$$
\begin{gathered}
\sum_{i=1}^{6} x_{i}=4 m^{2}-8 q^{2}, \\
\sum_{1 \leq i<j \leq 6} x_{i} x_{j}=22 q^{4}-4 m^{2} q^{2},
\end{gathered}
$$




$$
\begin{aligned}
\sum_{1 \leq i<j<k \leq 6} x_{i} x_{j} x_{k} & =-26 q^{6}, \\
\sum_{1 \leq i<j<k<l \leq 6} x_{i} x_{j} x_{k} x_{l} & =17 q^{8}, \\
\sum_{1 \leq i<j<k<l<p \leq 6} x_{i} x_{j} x_{k} x_{l} x_{p} & =-6 q^{10}, \\
\prod_{i=1}^{6} x_{i} & =q^{12} .
\end{aligned}
$$

Eliminating mass parameter, we obtain the mass-independent relation:

$$
\begin{aligned}
\sum_{1 \leq i<j \leq 6} x_{i} x_{j}+q^{2} \sum_{i=1}^{6} x_{i} & =14 q^{4}, \\
\sum_{1 \leq i<j<k \leq 6} x_{i} x_{j} x_{k} & =-26 q^{6}, \\
\sum_{1 \leq i<j<k<l \leq 6} x_{i} x_{j} x_{k} x_{l} & =17 q^{8}, \\
\sum_{1 \leq i<j<k<l<p \leq 6} x_{i} x_{j} x_{k} x_{l} x_{p} & =-6 q^{10}, \\
\prod_{i=1}^{6} x_{i} & =q^{12} .
\end{aligned}
$$

In terms of area $\mathscr{A}_{i}=4 \pi r_{i}^{2}=4 \pi x_{i}$ the above mass-independent relation could be written as

$$
\begin{aligned}
\sum_{1 \leq i<j \leq 6} \mathscr{A}_{i} \mathscr{A}_{j}+4 \pi q^{2} \sum_{i=1}^{6} \mathscr{A}_{i} & =14\left(4 \pi q^{2}\right)^{2}, \\
\sum_{1 \leq i<j<k \leq 6} \mathscr{A}_{i} \mathscr{A}_{j} \mathscr{A}_{k} & =-26\left(4 \pi q^{2}\right)^{3}, \\
\sum_{1 \leq i<j<k<l \leq 6} \mathscr{A}_{i} \mathscr{A}_{j} \mathscr{A}_{k} \mathscr{A}_{l} & =17\left(4 \pi q^{2}\right)^{4}, \\
\sum_{1 \leq i<j<k<l<p \leq 6} \mathscr{A}_{i} \mathscr{A}_{j} \mathscr{A}_{k} \mathscr{A}_{l} \mathscr{A}_{p} & =-6\left(4 \pi q^{2}\right)^{5}, \\
\prod_{i=1}^{6} \mathscr{A}_{i} & =\left(4 \pi q^{2}\right)^{6} .
\end{aligned}
$$

Again we have found mass-independent complicated function of horizon areas that could turn out to be universal in nature.

Case 3. Now we have set $\alpha=4$ and $\beta=6$. In this case the horizon equation is

$$
\begin{gathered}
r^{10}-2 m r^{9}+6 q^{2} r^{8}-6 m q^{2} r^{7}+12 q^{4} r^{6}-6 m q^{4} r^{5} \\
+11 q^{6} r^{4}-2 m q^{6} r^{3}+5 q^{8} r^{2}+q^{10}=0
\end{gathered}
$$

Again we apply Vieta's theorem; we get

$$
\begin{aligned}
\sum_{i=1}^{10} r_{i} & =2 m, \\
\sum_{1 \leq i<j \leq 10} r_{i} r_{j} & =6 q^{2}, \\
\sum_{1 \leq i<j<k \leq 10} r_{i} r_{j} r_{k} & =6 m q^{2}, \\
\sum_{1 \leq i<j<k<l \leq 10} r_{i} r_{j} r_{k} r_{l} & =12 q^{4}, \\
\sum_{1 \leq i<j<k<l<p \leq 10} r_{i} r_{j} r_{k} r_{l} r_{p} & =6 m q^{4}, \\
\sum_{1 \leq i<j<k<l<p<s \leq 10} r_{i} r_{j} r_{k} r_{l} r_{p} r_{s} & =11 q^{6}, \\
\sum_{1 \leq i<j<k<l<p<s<t<u \leq 10} r_{i<j<k<l<p<s<t \leq 10} r_{j} r_{k} r_{l} r_{p} r_{s} r_{t} & =2 m q^{6}, \\
\sum_{i=1} r_{j} r_{k} r_{l} r_{p} r_{s} r_{t} r_{u} & =5 q^{8}, \\
\prod_{i=1} r_{i} & =q^{10} .
\end{aligned}
$$

Eliminating mass parameter we have found the following set of mass-independent relation:

$$
\begin{aligned}
& 3 \sum_{1 \leq i<j<k<l<p<s<t \leq 10} r_{i} r_{j} r_{k} r_{l} r_{p} r_{s} r_{t} \\
& =q^{2} \sum_{1 \leq i<j<k<l<p \leq 10} r_{i} r_{j} r_{k} r_{l} r_{p}, \\
& \sum_{1 \leq i<j \leq 10} r_{i} r_{j}=6 q^{2}, \\
& \sum_{1 \leq i<j<k<l \leq 10} r_{i} r_{j} r_{k} r_{l}=12 q^{4},
\end{aligned}
$$

$\sum_{1 \leq i<j<k<l<p<s \leq 10} r_{i} r_{j} r_{k} r_{l} r_{p} r_{s}=11 q^{6}$,

$\sum_{1 \leq i<j<k<l<p<s<t<u \leq 10} r_{i} r_{j} r_{k} r_{l} r_{p} r_{s} r_{t} r_{u}=5 q^{8}$,

$\prod_{i=1}^{10} r_{i}=q^{10}$ 
If we are working in terms of area then the above massindependent relation could be written as

$$
\begin{aligned}
& 3 \sum_{1 \leq i<j<k<l<p<s<t \leq 10} \sqrt{\mathscr{A}_{i} \mathscr{A}_{j} \mathscr{A}_{k} \mathscr{A}_{l} \mathscr{A}_{p} \mathscr{A}_{s} \mathscr{A}_{t}} \\
& =4 \pi q^{2} \sum_{1 \leq i<j<k<l<p \leq 10} \sqrt{\mathscr{A}_{i} \mathscr{A}_{j} \mathscr{A}_{k} \mathscr{A}_{l} \mathscr{A}_{p}}, \\
& \sum_{1 \leq i<j \leq 10} \sqrt{\mathscr{A}_{i} \mathscr{A}_{j}}=24 \pi q^{2} \\
& \sum_{1 \leq i<j<k<l \leq 10} \sqrt{\mathscr{A}_{i} \mathscr{A}_{j} \mathscr{A}_{k} \mathscr{A}_{l}}=3\left(8 \pi q^{2}\right)^{2} \\
& \sum_{1 \leq i<j<k<l<p<s \leq 10} \sqrt{\mathscr{A}_{i} \mathscr{A}_{j} \mathscr{A}_{k} \mathscr{A}_{l} \mathscr{A}_{p} \mathscr{A}_{s}}=11\left(4 \pi q^{2}\right)^{3}, \\
& \sum_{1 \leq i<j<k<l<p<s<t<u \leq 10} \sqrt{\mathscr{A}_{i} \mathscr{A}_{j} \mathscr{A}_{k} \mathscr{A}_{l} \mathscr{A}_{p} \mathscr{A}_{s} \mathscr{A}_{t} \mathscr{A}_{u}} \\
& =5\left(4 \pi q^{2}\right)^{4}, \\
& \prod_{i=1}^{10} \sqrt{\mathscr{A}_{i}}=\left(4 \pi q^{2}\right)^{5}
\end{aligned}
$$

$$
C_{i}=\frac{\partial m}{\partial T_{i}}
$$

For generalized regular $\mathrm{BH}$, it is found to be

$$
C_{i}=\frac{\left(\partial m / \partial r_{i}\right)}{\left(\partial T_{i} / \partial r_{i}\right)}
$$

where

It should be noted that the Hawking temperature product depends on the mass parameter and thus it is not a universal uantity. that determines the thermodynamic stability of $\mathrm{BH}$ is defined by

$$
\begin{aligned}
T_{i} & =\frac{\kappa_{i}}{2 \pi}=\frac{1}{4 \pi}\left[\alpha \frac{r_{i}}{\left(r_{i}^{2}+q^{2}\right)}-\frac{(\alpha-1)}{r_{i}}\right. \\
& \left.+\frac{q^{2}(\alpha-\beta) r_{i}^{\beta-1}}{\left(r_{i}^{2}+q^{2}\right)^{\beta / 2+1}}+\frac{q^{2}(\beta-\alpha-1) r_{i}^{\beta-2}}{\left(r_{i}^{2}+q^{2}\right)^{\beta / 2}}\right] .
\end{aligned}
$$

turn at could thermodynamic product relations for different values of $\alpha$ nd $\beta$ which are mass-independent.

The Hawking [18] temperature on $\mathscr{H}^{i}$ reads off

$$
\begin{aligned}
\frac{\partial m}{\partial r_{i}}= & \frac{\left[\alpha r_{i}^{\alpha}\left(r_{i}^{2}+q^{2}\right)^{\alpha / 2-1}-(\alpha-1) r_{i}^{\alpha-2}\left(r_{i}^{2}+q^{2}\right)^{\alpha / 2}\right]}{2 r_{i}^{2(\alpha-1)}} \\
& +\frac{q^{2}\left[(\beta-\alpha-1) r_{i}^{\beta-\alpha-2}\left(r_{i}^{2}+q^{2}\right)^{(\beta-\alpha) / 2}-(\beta-\alpha) r_{i}^{\beta-\alpha}\left(r_{i}^{2}+q^{2}\right)^{(\beta-\alpha-2) / 2}\right]}{\left(r_{i}^{2}+q^{2}\right)^{\beta-\alpha}}, \\
\frac{\partial T_{i}}{\partial r_{i}}= & \frac{1}{4 \pi}\left[\frac{\alpha-1}{r_{i}^{2}}-\frac{\alpha\left(r_{i}^{2}-q^{2}\right)}{\left(r_{i}^{2}+q^{2}\right)^{2}}+q^{2}(\alpha-\beta) \frac{\left\{(\beta-1) r_{i}^{\beta-2}\left(r_{i}^{2}+q^{2}\right)^{\beta / 2+1}-(\beta+2) r_{i}^{\beta}\left(r_{i}^{2}+q^{2}\right)^{\beta / 2}\right\}}{\left(r_{i}^{2}+q^{2}\right)^{\beta+2}}\right] \\
& +\frac{1}{4 \pi}\left[q^{2}(\beta-\alpha-1) \frac{\left\{(\beta-2) r_{i}^{\beta-3}\left(r_{i}^{2}+q^{2}\right)^{\beta / 2}-\beta r_{i}^{\beta-1}\left(r_{i}^{2}+q^{2}\right)^{\beta / 2-1}\right\}}{\left(r_{i}^{2}+q^{2}\right)^{\beta}}\right] .
\end{aligned}
$$


The $\mathrm{BH}$ undergoes a second-order phase transition when $\partial T_{i} / \partial r_{i}=0$. In this case the specific heat diverges.

Finally, Komar [21] energy computed at $\mathscr{H}^{i}$ is given by

$$
\begin{aligned}
E_{i} & =\frac{1}{2}\left[\alpha \frac{r_{i}^{3}}{\left(r_{i}^{2}+q^{2}\right)}-(\alpha-1) r_{i}+\frac{q^{2}(\alpha-\beta) r_{i}^{\beta+1}}{\left(r_{i}^{2}+q^{2}\right)^{\beta / 2+1}}\right. \\
& \left.+\frac{q^{2}(\beta-\alpha-1) r_{i}^{\beta}}{\left(r_{i}^{2}+q^{2}\right)^{\beta / 2}}\right] .
\end{aligned}
$$

In the limits $\alpha=3$ and $\beta=4$, one obtains the result of $A B G$ $\mathrm{BH}$.

\section{Discussion}

In this work, we examined thermodynamic product relations for generalized regular (curvature-free) $\mathrm{BH}$. Generalized $\mathrm{BH}$ means the $\mathrm{BHs}$ represented by the four parameters, that is, $m, q, \alpha$, and $\beta$. We determined different thermodynamic product particularly area products for different values of $\alpha$ and $\beta$. We showed that there is some complicated function of horizon areas indeed mass-independent that could turn out to be universal. We also derived the specific heat to determine the thermodynamic stability of the BH. Finally, we computed Komar energy for this generalized $\mathrm{BH}$.

\section{Competing Interests}

The author declares that they have no competing interests.

\section{References}

[1] M. Ansorg and J. Hennig, "Inner Cauchy horizon of axisymmetric and stationary black holes with surrounding matter in Einstein-Maxwell theory," Physical Review Letters, vol. 102, no. 22, Article ID 221102, 2009.

[2] M. Visser, "Area products for stationary black hole horizons," Physical Review D, vol. 88, Article ID 044014, 2013.

[3] J. Hennig, "Geometric relations for rotating and charged AdS black holes," Classical and Quantum Gravity, vol. 31, no. 13, Article ID 135005, 9 pages, 2014.

[4] P. Pradhan, "Area (or entropy) product formula for a regular black hole," General Relativity and Gravitation, vol. 48, no. 2, article 19, 11 pages, 2016.

[5] M. Cvetič, G. W. Gibbons, and C. N. Pope, "Universal area product formulas for rotating and charged black holes in four and higher dimensions," Physical Review Letters, vol. 106, no. 12, Article ID 121301, 4 pages, 2011.

[6] A. Castro and M. J. Rodriguez, "Universal properties and the first law of black hole inner mechanics," Physical Review D, vol. 86, no. 2, Article ID 024008, 5 pages, 2012.

[7] S. Detournay, "Inner mechanics of three-dimensional black holes," Physical Review Letters, vol. 109, no. 3, Article ID 031101, 5 pages, 2012.

[8] D. N. Page and A. A. Shoom, "Universal area product for black holes: a heuristic argument," Physical Review D, vol. 92, no. 4, Article ID 044039, 2015.

[9] P. Pradhan, "Black hole interior mass formula," The European Physical Journal C, vol. 74, no. 5, article 2887, 2014.
[10] P. Pradhan, "Thermodynamic product formula for HořavaLifshitz black hole," Physics Letters B, vol. 747, pp. 64-67, 2015.

[11] E. Ayón-Beato and A. García, "Four-parametric regular black hole solution," General Relativity and Gravitation, vol. 37, no. 4, pp. 635-641, 2005.

[12] N. Bretón, "Smarr's formula for black holes with non-linear electrodynamics," General Relativity and Gravitation, vol. 37, no. 4, pp. 643-650, 2005.

[13] S. Bose and N. Dadhich, "Brown-York quasilocal energy, gravitational charge, and black hole horizons," Physical Review D, vol. 60, no. 6, Article ID 064010, 7 pages, 1999.

[14] L. Balart, "Quasilocal energy, Komar charge and horizon for regular black holes," Physics Letters B, vol. 687 , no. $4-5$, pp. 280 285, 2010.

[15] S. Ansoldi, "Spherical black holes with regular center: a review of existing models including a recent realization with Gaussian sources," https://arxiv.org/abs/0802.0330.

[16] M. S. Ma and R. Zhao, "Corrected form of the first law of thermodynamics for regular black holes," Classical and Quantum Gravity, vol. 31, no. 24, Article ID 245014, 2014.

[17] J. D. Bekenstein, "Black holes and entropy," Physical Review D, vol. 7, pp. 2333-2346, 1973.

[18] J. M. Bardeen, B. Carter, and S. W. Hawking, "The four laws of black hole mechanics," Communications in Mathematical Physics, vol. 31, pp. 161-170, 1973.

[19] E. Ayón-Beato and A. García, "Regular black hole in general relativity coupled to nonlinear electrodynamics," Physical Review Letters, vol. 80, no. 23, pp. 5056-5059, 1998.

[20] J. M. Bardeen, in Conference Proceedings of GR5, U.S.S.R, Tbilisi, Georgia, 1968.

[21] A. Komar, "Covariant conservation laws in general relativity," Physical Review Letters, vol. 113, no. 3, pp. 934-936, 1959. 

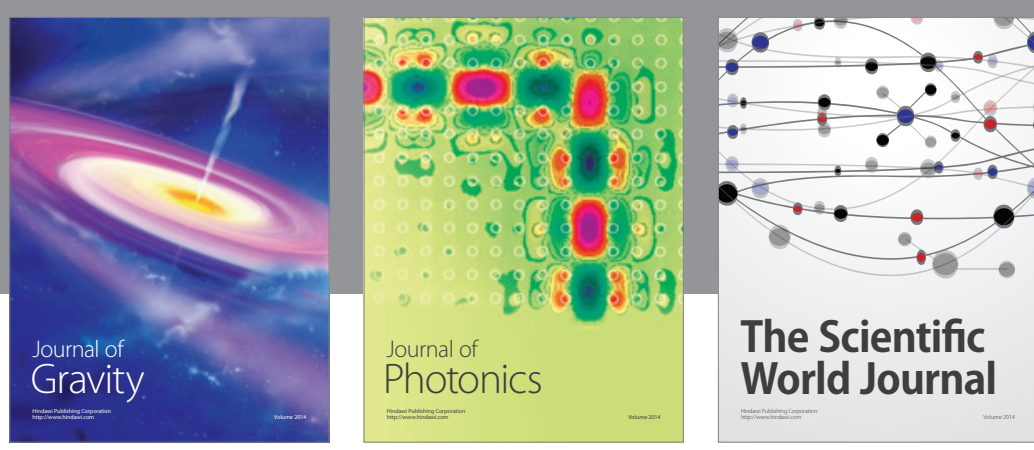

The Scientific World Journal
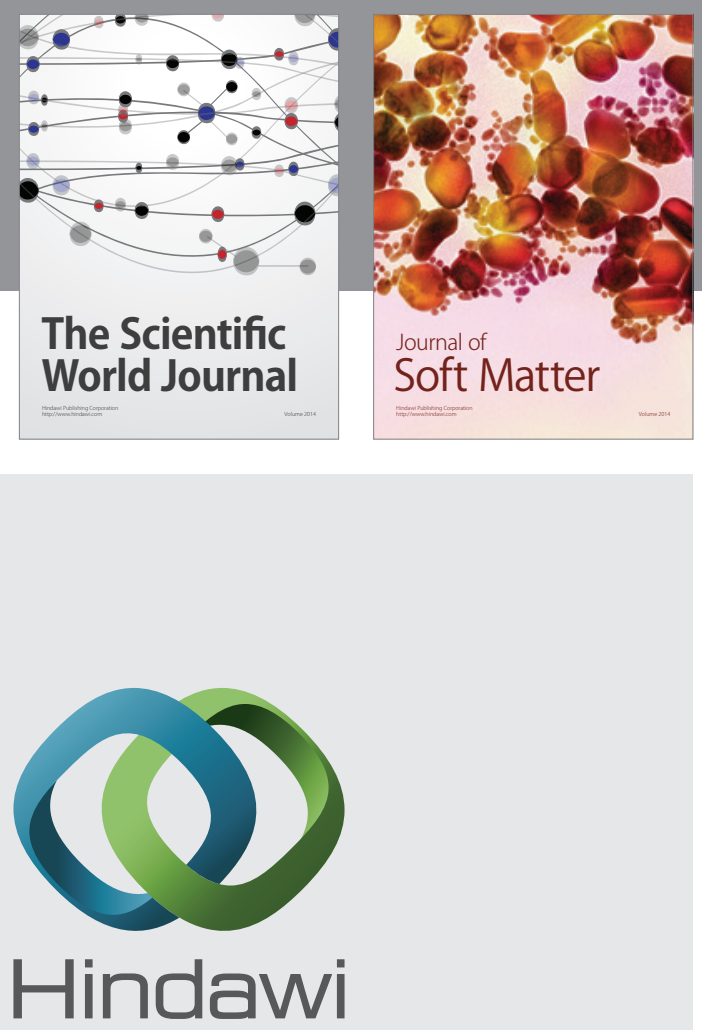

Submit your manuscripts at

http://www.hindawi.com

nternational Journal of

Statistical Mechanics
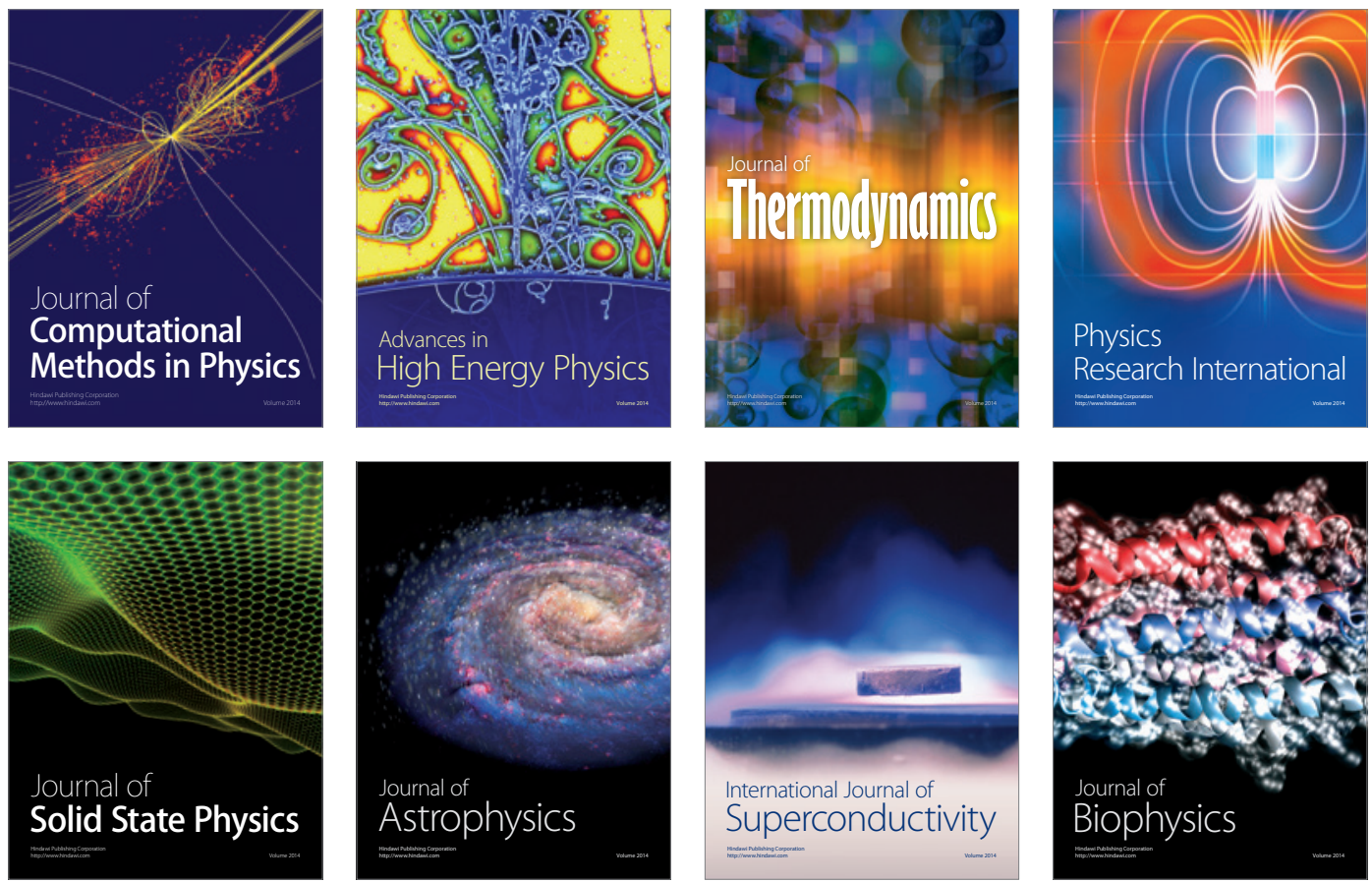
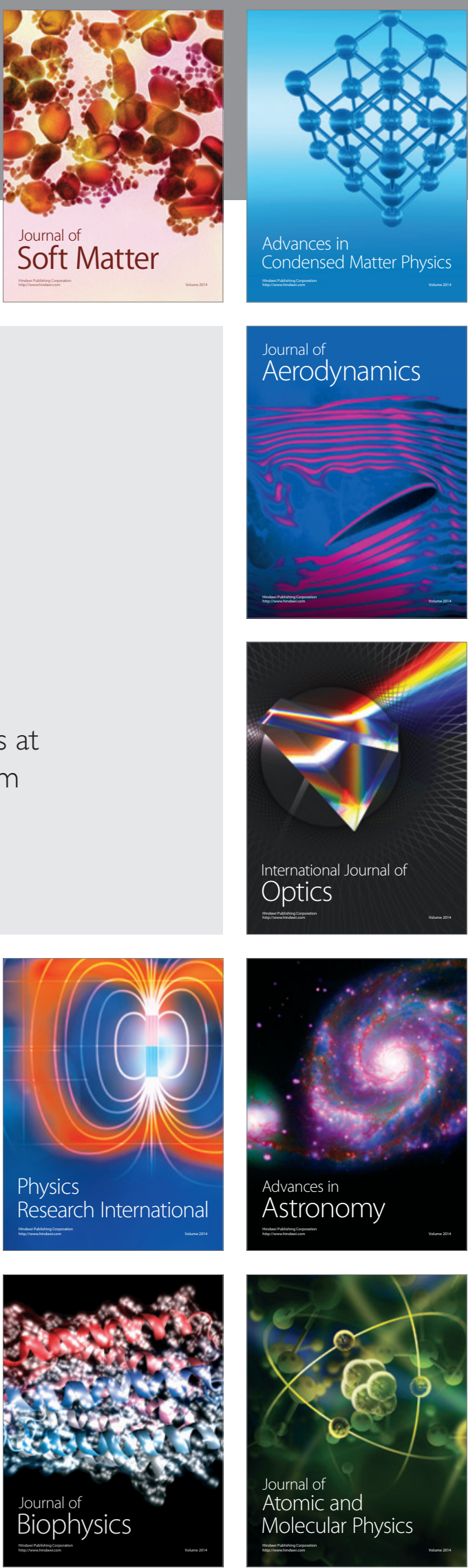